\title{
The Evolution of Molecular Clouds
}

\author{
Richard B. Larson
}

Yale Astronomy Department, Box 6666, New Haven, CT 06511, U.S.A.

\section{Introduction}

Molecular clouds constitute the densest parts of the interstellar medium in galaxies, and ever since their discovery 25 years ago they have been of prime interest as the sites of star formation. Molecular clouds also play an important galactic role in that massive stars recently formed in them provide the main energy sources for the interstellar medium, partly by destroying their birth clouds and recycling their matter back into more diffuse forms. Thus an understanding of the life cycle of molecular clouds is of central importance not only for understanding how stars form, but also for understanding the dynamics of the interstellar medium and ultimately the evolution of galaxies as a whole. Many complex processes are clearly involved, most of which are not yet well understood; however, the increasingly detailed observational information that is becoming available about molecular clouds and star formation is beginning to allow us at least to identify some of the most important processes at work. In this contribution, I shall review briefly some relevant basic characteristics of molecular clouds and some of the many processes that are likely to play a role in their formation, evolution, and destruction.

\section{Basic Cloud Characteristics}

One fundamental characteristic of molecular clouds is that they are not, as has sometimes been assumed, isolated 'billiard balls' moving about independently in space, but instead are just dense condensations in more widely distributed, mostly atomic gas. Although molecular clouds may often appear to have sharp boundaries, these boundaries do not represent the edge of the matter distribution but just rapid transitions from the molecular gas to the surrounding atomic gas, which is distributed in extended envelopes that typically have comparable mass (Blitz 1988, 1991). The molecular composition of interstellar clouds depends on the local gas density $n$ and on the column density $N$ of material providing shielding from dissociating ultraviolet radiation, and the gas is predicted to be predominantly molecular wherever the product $n N^{2 / 3}$ exceeds a critical value that depends on the ultraviolet radiation flux and the dust abundance (Elmegreen 1989, 1993). Since the condition required for the gas in an interstellar cloud to be mainly molecular is independent of the condition for self-gravity to be important, there is no reason why molecular clouds should necessarily be gravitationally bound, or vice versa; the molecular content of a cloud or region could even change rapidly with time because of its sensitivity to local conditions 
such as the radiation field that can vary with time (Elmegreen 1993). Thus, there need be no close correspondence between the amount of molecular gas in a region and the amount of star formation occurring there, and indeed it is found that star formation rates in galaxies correlate better with the total gas content than with the content of molecular gas alone (Buat, Deharveng, \& Donas 1989; Kennicutt 1989, 1990; Evans 1991). The Small Magellanic Cloud is an example of an actively star-forming system in which only a small fraction of the interstellar medium is molecular, owing to a high ultraviolet flux and a low dust abundance (Rubio, Lequeux, \& Boulanger 1993).

Another important characteristic of molecular clouds is that they are transient structures and do not survive without major changes for more than a few times $10^{7}$ years (Blitz \& Shu 1980; Larson 1981). The short lifetimes of molecular clouds are directly indicated by the fact that the range in age of the young stars associated with them is only about 10 to $20 \mathrm{Myr}$, comparable to the internal dynamical timescales of large molecular clouds (Larson 1981); age spans of this order are found both for the T Tauri stars in dark clouds (Cohen \& Kuhi 1979) and for the subgroups of OB stars in more massive star-forming complexes (Blaauw 1964, 1991). Subgroups of OB stars whose ages are greater than 10 Myr no longer contain significant amounts of gas or dust, so the gas from which such groups form is evidently cleared away in a time of this order (Blaauw 1991); those clouds or parts of clouds that form star clusters are also dispersed in a similar time, since little or no molecular gas is seen within $25 \mathrm{pc}$ of clusters older than 10 Myr (Bash, Green, \& Peters 1977; Leisawitz, Bash, \& Thaddeus 1989). There cannot be any long 'dead time' between the formation of a massive molecular cloud and the onset of star formation in it, since very few of the largest molecular clouds are not forming stars (Blitz 1991), and the number of molecular clouds of all masses that are not forming stars is only about comparable to the number of star-forming clouds (Mooney \& Solomon 1988; Solomon, this conference). Therefore the total lifetimes of molecular clouds, or at least of those parts of them that form systems of stars, cannot exceed twice the period of active star formation in them, or perhaps $\sim 20$ Myr (see also Elmegreen 1991a, who estimates a cloud lifetime of $\sim 40$ Myr from similar arguments). Thus there is no need, as has often been supposed, for molecular clouds to be supported against gravity in a quasi-equilibrium configuration for many dynamical times. While it is true that virial balance is often roughly satisfied, at least in the larger clouds (Larson 1981), this does not rule out the possibility of rapid dynamical evolution of these clouds, or of those parts of them that form groups or clusters of stars.

Additional evidence that molecular clouds are short-lived, or at least that their structures change rapidly with time, is provided by the fact that they appear to be chemically relatively unevolved; that is, the abundances of various molecules are often far from those expected to prevail in chemical equilibrium, and resemble instead those predicted to occur at an early stage of chemical processing less than 1 Myr after cloud formation (Prasad et al. 1987; Herbst \& Leung 1989; Herbst, this conference). It has also been a long-standing problem to understand why most of the molecules in dense cold molecular clouds have not frozen out on dust grains, as would be expected if these clouds are stable long-lived objects. A number of authors have therefore suggested that molecular 
clouds are actually relatively young (Stahler 1984), or that they experience rapid dynamical evolution that leads to frequent chemical reprocessing of their gas, for example by turbulent cycling between the interiors and the surfaces of clouds (Chièze \& Pineau des Forêts 1990) or by the frequent formation and dispersal of dense clumps in them (Prasad 1991).

A third notable property of molecular clouds is that they are highly irregular structures and have complex shapes that do not at all resemble equilibrium configurations. Many of them have wispy or filamentary shapes resembling those of atmospheric clouds, as is well illustrated in the IRAS 100-micron maps of molecular clouds (Scalo 1990; Boulanger 1991). The atmospheric clouds with wispy structures are transient and are typically dispersing rapidly, and this may be true also of many molecular clouds, especially the smaller and more diffuse ones which are mostly not gravitationally bound (Herbertz, Ungerechts, \& Winnewisser 1991; Falgarone, Puget, \& Pérault 1992). The irregular boundaries of molecular clouds on contour maps have been found by a number of authors to have fractal properties (Scalo 1990; Dickman, Horvath, \& Margulis 1990; Falgarone, Phillips, \& Walker 1991; Falgarone 1992; Zimmermann \& Stutzki 1992), and the fractal dimension inferred for the surfaces of these clouds is intriguingly similar to the fractal dimension that has been found for various interfaces in turbulent flows (Falgarone \& Phillips 1991; Sreenivasan 1991). Although the significance of this result is not presently clear, it seems at least consistent with the possibility that molecular clouds are condensations in a turbulent medium and are structured partly by turbulence (Larson 1979, 1981; Falgarone \& Phillips 1991). The possibility that turbulence plays an important role in determining cloud properties is supported by the fact that the basic scaling relations satisfied by molecular clouds, such as the size-linewidth relation, are similar for both bound and unbound clouds, and are similar also to those expected for turbulent flows (Falgarone et al. 1992; Falgarone 1992). It is clear in any case that simple models cannot provide an adequate description of the structure or dynamics of molecular clouds. Models incorporating a realistic degree of structural complexity, such as fractal models, may for some purposes provide a more useful description (e.g. Hetem \& Lépine 1993).

\section{Cloud Formation}

Since molecular clouds are transient features, it follows that they are constantly being formed and destroyed. Therefore, it is necessary to understand the processes by which they are continually being reassembled from more dispersed gas. The rate at which interstellar gas is presently being collected into star-forming molecular clouds in our Galaxy is related to the star formation rate, and it can be estimated empirically from the observed star formation rate and the efficiency of star formation in molecular clouds. The total rate of star formation in our Galaxy is of the order of $3 \mathrm{M}_{\odot}$ per year, and since only about 2 percent or less of the mass of a typical molecular cloud is converted into stars (Myers et al. 1986; Leisawitz et al. 1989; Evans \& Lada 1991), this implies that at least $150 \mathrm{M}_{\odot}$ of gas per year is being turned into star-forming molecular clouds. Since the total amount of gas in our Galaxy is about $5 \times 10^{9} \mathrm{M}_{\odot}$, the average time required to 
collect gas into giant molecular clouds must then be about 30 Myr. A similar estimate for the solar neighborhood, where the timescales are somewhat longer than the Galactic average, yields a formation timescale for molecular clouds of about $50 \mathrm{Myr}$ (Larson 1991a, 1992b). Note that this estimated formation time is not much longer than the cloud lifetime of $\sim 20 \mathrm{Myr}$ (Section 2); thus the formation of molecular clouds must itself be a rather rapid process, and cannot take many dynamical timescales. Since the timescales for the formation, internal evolution, and destruction of molecular clouds are all of the same order, these processes probably cannot be clearly separated in time, and they may all go on simultaneously in different parts of a star-forming complex.

Two possible formation mechanisms for molecular clouds that have been considered in some detail are (1) cloud growth by random collisions and coalescence, and (2) gravitational instability or 'swing amplification' effects in the Galactic gas layer; these and other mechanisms have been discussed by Larson (1987, 1988, 1992b), and in more detail by Elmegreen (1990a,b, 1991a,b). The first possibility, i.e. the building of large clouds from smaller ones by random collisions and coalescence, predicts formation times of at least $100 \mathrm{Myr}$ for giant molecular clouds, and therefore probably cannot be the primary formation mechanism because this is longer than the cloud formation timescale estimated empirically; in any case, most collisions between smaller clouds are probably disruptive and so are not likely to result in coalescence. The second possible mechanism, i.e. large-scale gravitational instability and amplification effects in the Galactic gas layer, is almost certainly more important because it can collect gas into large complexes on a timescale that is only about $40 \mathrm{Myr}$ in the solar neighborhood, in good agreement with the estimated cloud formation time. Evidence that gravitational instability effects are indeed primarily responsible for both molecular cloud formation and star formation in galaxies is provided by the fact that star formation is observed to occur only where the surface density of gas in galactic disks exceeds a threshold which is close to the critical value predicted for the onset of gravitational instability (Kennicutt 1989, 1990).

A true instability with unlimited growth of density perturbations probably does not occur in most galaxies, since the stability parameter $Q$ is typically somewhat larger than unity and so is in the stable regime; nevertheless, limited 'swing amplification' of shearing density perturbations can still occur in this situation (Toomre 1981), and this is probably the effect mostly responsible for collecting interstellar gas into large star-forming cloud complexes and spiral arm segments in galaxies. The threshold gas surface density found by Kennicutt $(1989,1990)$ to be required for star formation actually corresponds to a value for $Q$ of about 1.7, approximately the value below which swing amplification becomes important (Larson 1992b), thus supporting the relevance of this mechanism for star formation. Density waves may of course also play a role in driving cloud formation and star formation in galaxies, but their presence is not necessary, as is shown by the example of the irregular galaxies, and it may be that in many cases the role of density waves is largely just one of organizing star formation that would have occurred anyway (Elmegreen 1992).

The formation of massive molecular clouds by gravitational instability or swing amplification effects in a turbulent interstellar medium must also involve complex smaller-scale processes, including collisions between the small clouds 
that were present in the initial medium and the building up of larger clouds by accretion processes of some kind. The possible role of gas accumulation processes in the internal evolution of molecular clouds has been discussed by Larson (1982) and Myers (1991), and simulations of cloud collisions and coalescence in a contracting cloud complex have been carried out by Monaghan \& Varnas (1988). While collisions almost certainly play a role in the building up of large molecular clouds, it nevertheless seems clear that purely random collisions cannot build them fast enough and that more ordered large-scale motions are therefore required, such as those that are involved in the formation of large cloud complexes or spiral arm segments in galaxies. This conclusion is, of course, consistent with the fact that most of the star formation in galaxies is observed to occur in large complexes or spiral arm segments (Efremov 1989).

\section{Cloud Collapse and Fragmentation}

As has been noted, a giant molecular cloud must begin forming stars soon after the cloud itself has formed, since relatively few of the largest molecular clouds are not forming stars. Even if as many as half of all molecular clouds are not forming stars (Solomon, this conference), the time delay between the formation of a molecular cloud and the onset of star formation in it cannot exceed the subsequent duration of the star formation activity, which is of the order of 10 Myr and comparable to the internal dynamical timescale (Section 2). Since it takes somewhat longer than this to build large molecular clouds, it is likely that star formation begins already in molecular clouds while they are still being assembled; moreover, star formation must begin within a time not much longer than the dynamical or free-fall time of such a cloud. Collapse and star formation can occur in the densest part of a cloud even if the cloud as a whole is not collapsing, and this must in fact be what usually happens because there is no evidence that most star-forming clouds are undergoing any rapid overall collapse, and there is even evidence that many of them are being dispersed, as will be discussed in Sections 5 and 6 .

According to the classical picture, star formation involves the collapse of a cloud or part of a cloud under gravity and the associated fragmentation of the cloud into smaller and smaller bound clumps; this is expected to occur because molecular clouds typically contain many times the 'Jeans mass', which is the minimum mass for gravitationally bound fragments. It is possible, however, that much of the small-scale structure that eventually develops into stars and groups of stars is present from the beginning because star-forming clouds are assembled from gas that already has much small-scale structure. There is as yet little direct evidence bearing on this question, but one possible piece of evidence that fragmentation does occur in star-forming clouds, at least in the sense that small-scale density fluctuations are strongly amplified with time, is provided by the fact that one of the few large molecular clouds that is not presently forming stars contains relatively few small clumps compared with clouds that are forming stars (Williams \& Blitz 1993); if this cloud is at an early stage of evolution and will later evolve to a star-forming stage, this suggests that many more small clumps will be formed in this process. 
For a variety of reasons, including the effects of initial asymmetries, magnetic fields, and turbulence, the collapse of the densest parts of molecular clouds will almost certainly not be spherical but will tend to produce flattened or filamentary structures (Larson 1985; Miyama, Narita, \& Hayashi 1987a,b). Even if flattened structures are the first to be formed, for example by collapse along magnetic field lines, they may tend to break up into filaments before finally fragmenting into clumps (Miyama et al. 1987a,b); there is in any case abundant evidence that molecular clouds often have elongated or filamentary shapes (Schneider \& Elmegreen 1979; Scalo 1990; Myers et al. 1991; Bally et al. 1991). Most numerical simulations of collapse and fragmentation have modeled the idealized case of an initially nearly uniform, spherical, rapidly rotating cloud that collapses first to a disk and then fragments into clumps (Larson 1978; Miyama, Hayashi, \& Narita 1984; Monaghan \& Lattanzio 1991). The typical masses of the clumps that form are comparable to the Jeans mass calculated for a fragmenting gas layer, and with typical molecular cloud temperatures and column densities, this mass is of the order of one solar mass (Larson 1985). The numerical studies quoted above also illustrate in increasing detail the tendency for fragmenting disks to develop filamentary and even spiral small-scale structures before most of the gas condenses into clumps; such spiral structure plays an important role in the redistribution of angular momentum by gravitational torques that must occur if most of the mass is to end up in condensed objects (Larson 1984).

The above calculations may not, however, be very relevant to real molecular clouds, most of which are far from spherical and are rotating only rather slowly (Goodman et al. 1993). The probably more realistic case of the collapse of an elongated cylindrical cloud has been studied numerically by Bastien et al. (1991) and Arcoragi et al. (1991), and the effect of including a small amount of rotation has been studied by Bonnell et al. (1991, 1992) and Bonnell \& Bastien (1992). A cylindrical cloud tends to collapse first toward its axis to form a thin filament, and it then fragments along its length into two or more dense clumps (Larson 1972). The masses of the clumps are similar in order of magnitude to the Jeans mass for a fragmenting cylinder, but the tendency of the cloud to collapse first to a thin filament results in somewhat smaller fragments which may have masses as small as one-quarter of the initial Jeans mass (Bastien et al. 1991), possibly accounting for the formation of stars with masses as small as $\sim 0.1$ solar masses (Larson 1992a).

Qualitatively new results are found when the initially cylindrical cloud is given a slow rotation about an arbitrary axis: the fragments then develop surrounding disks, and these disks may fragment further and may also interact with each other as the fragments fall closer together, leading eventually to the formation of binary and multiple systems with a variety of properties (Bonnell et al. 1991, 1992). Somewhat similar results have been found by Boss (1993) and by Burkert \& Bodenheimer (1993), who have calculated the collapse of initially spherical rotating clouds with bar-like density perturbations; in some cases these clouds collapse to elongated filaments that fragment along their lengths into two or more objects. Such phenomena may well account for the origin of many of the observed binary and multiple systems. Protostellar interactions involving disks can also have a number of interesting consequences, including the tilting of the disks and the stripping of mass from them, as well as the formation of 
additional binary systems by capture and the modification of binary orbits by gravitational drag effects (Herbig \& Terndrup 1986; Larson 1990; Pringle 1991; Heller 1993a,b,c). Such effects could be especially important if stars form in dense clusters, as appears to be the case for most stars (see Section 5).

Direct observational evidence for collapse and fragmentation processes has proven difficult to find. One reason for this is the well-known fact that stars generate vigorous outflows as soon as they are formed, and these outflows tend to dominate the observed properties of star-forming regions and to obscure the relatively modest expected infall motions. The collapse of a dense protostellar clump into a single star or small group of stars would in any case be difficult to observe because measurable infall velocities are expected only in a very small region. Collapse might be easier to observe if a massive molecular cloud core collapses to form an entire cluster of stars, since larger infall velocities could then be produced over a larger region of space. If a newly formed cluster is observed at a sufficiently early time, the effects of the young stars on their environment might still remain confined to a small region and infall motions might still be observable outside this region. A possible example of this is provided by the core of the W49A molecular cloud, which contains a compact group of newly formed $\mathrm{O}$ stars surrounded by ultracompact $\mathrm{H}$ II regions (Welch et al. 1987). The molecular line profiles observed toward these $\mathrm{H}$ II regions have redshifted absorption components attributable to infalling gas on the near side of the cluster, and the inferred infall velocity of $\sim 10 \mathrm{~km} / \mathrm{s}$ is comparable to the predicted free-fall velocity of the massive W49A cloud core. Further possible examples of the infall of residual cloud material toward newly formed clusters of massive stars have been described by Welch (1993; this conference). In all of these cases, as with the nearer and more extensively studied examples of cluster formation discussed below, the timescale for the collapse of the cloud core and the formation of the associated cluster appears to be quite short, i.e. of the order of $1 \mathrm{Myr}$ or less, suggesting that the evolution of the densest part of a molecular cloud can be quite rapid and can approach a free-fall collapse.

\section{Formation of Star Clusters}

The numerical simulations discussed above suggest that stars may generally form in multiple systems or groups of some sort, possibly hierarchical in structure, but simulations of the formation of multiple stellar systems are still at an early stage, and simulations of the formation of an entire cluster of stars are not yet feasible owing to the complexity of the processes involved. Thus, theoretical work cannot yet address many of the details of how systems of stars form. However, observational studies of star formation have made great strides in recent years, thanks largely to new instrumentation such as infrared cameras and improved millimeter-wavelength telescopes and interferometers, and the resulting data are beginning to provide the basis for a phenomenological approach to understanding of the formation of systems of stars. Infrared cameras have, in particular, made it possible to survey large areas of star-forming clouds for newborn stars still embedded in them, and a major discovery has been the finding that most of the recently formed stars in nearby molecular clouds are located in compact 
clusters associated with massive dense cloud cores; it thus appears that the formation of compact clusters of stars is the dominant mode of star formation, at least in well-studied nearby star-forming regions (Lada \& Lada 1991; Lada 1992; Carpenter et al. 1993; McCaughrean 1993; Lada, Strom, \& Myers 1993; Zinnecker, McCaughrean, \& Wilking 1993). The isolated mode of star formation that has so far received the most theoretical attention may therefore not be relevant for most stars, and strongly clustered star formation and processes such as the protostellar interactions mentioned above may be more important than has previously been recognized.

Even in the Taurus-Auriga dark clouds, which have usually been regarded as exemplifying an isolated mode of star formation (e.g., Lada et al. 1993), most of the young stars are actually not completely isolated but are concentrated in small groupings associated with the most prominent dark clouds (Herbig 1977; Larson 1982; Myers 1987). Gomez et al. (1993) have analyzed the spatial distribution of all of the known young stars in the Taurus-Auriga region, and have found evidence for clustering on all scales from 0.005 pc to 5 pc; in particular, they have found that the two-point angular correlation function for these stars is approximately a power-law function of separation over this range of distances, suggesting that completely isolated star formation is rare even in the TaurusAuriga clouds, and also that similar mechanisms may be operating over a wide range of length scales to produce the observed approximately scale-free clustering. One possible way of producing such scale-free clustering might be that gravitational drag or 'dynamical friction' effects, which have no preferred length scale, act on dense protostellar clumps or forming stars in a collapsing and fragmenting cloud and cause them to become increasingly clustered, possibly in a hierarchical fashion (Larson 1990, 1991b).

The nearest newly formed star cluster is the compact grouping of more than 100 infrared stellar objects embedded in the dense core of the $\rho$ Ophiuchi molecular cloud (Wilking 1992; Greene \& Young 1992; Lada et al. 1993). This cluster contains most of the young stars known to be associated with the $\rho$ Oph cloud, and it is also the most active current site of star formation in the entire Ophiuchus-Scorpius-Centaurus region, in which several earlier episodes of star formation have produced OB associations or subgroups (Blaauw 1991). It is thus of considerable interest to examine the circumstances in which the $\rho$ Oph cluster has formed, since they may yield clues to the processes involved. The CO survey of this region by de Geus, Bronfman, \& Thaddeus (1990) shows that the $\rho$ Oph cloud core and its embedded cluster are located in the head of a comet-shaped cloud closely adjacent to or partly within the Upper Scorpius OB association, and that this cloud and several others in its vicinity have long tails that point radially away from the center of this association. The windswept appearance of these clouds strongly suggests that they have been shaped by outflows from the OB association, perhaps in somewhat the same way that comets are shaped by the solar wind. The explosive effects of outflows or pressures generated by the OB association on the surrounding interstellar medium are clearly demonstrated by the presence of a large cavity in the distribution of the atomic gas in this region, bounded by a shell that appears to be expanding away from the OB association (de Geus 1991; Blaauw 1991). The molecular clouds in this region 
mostly appear to have been formed, or at least compressed, where this shell interacts with the denser atomic gas closer to the Galactic plane.

Similar phenomena appear to be occurring on a larger scale in the Orion region, especially in the Orion A cloud which contains the prominent and highly concentrated Trapezium cluster of more than 500 young stars partially embedded in the dense OMC1 core region of the cloud (Herbig \& Terndrup 1986; McCaughrean 1989; McCaughrean, Rayner, \& Zinnecker 1991; Zinnecker et al. 1993). The Trapezium cluster is closely associated with, and appears to have formed from, a dense clumpy molecular filament that contains three currently active sites of star formation, including the luminous and intensively studied IRc2 region (Hasegawa 1987; Sargent \& Mundy 1988; Genzel \& Stutzki 1989). This dense $\int$-shaped filament or 'ridge' of molecular gas is also the dominant feature in the head of the strikingly comet-like and filamentary Orion A cloud (Bally et al. 1987, 1991). As is the case with the $\rho$ Oph cloud, the Orion A cloud is located closely adjacent to an $\mathrm{OB}$ association, and its long tail points away from the center of the association; again, the structure of this cloud strongly suggests that it has been shaped and compressed by outflows or pressures from the OB association (Bally et al. 1987, 1991). The cometary shape of the Orion A cloud, and even the dense filament in its head, are reminiscent of the results of Woodward's (1978) simulation of the deformation of an interstellar cloud swept over by a shock front in the intercloud medium; most of the material in this cloud is ablated away into a long 'comet tail', but a small part of it is compressed into a dense elongated clump in the head of the comet.

The adjacent Orion B cloud also borders on the Orion OB association, and it also shows evidence of being compressed by outflows from the association: the edge of this cloud nearest the center of the association is relatively sharply bounded and contains several dense clumps (Blaauw 1991; Bally et al. 1991), most of which again contain embedded clusters of young stars similar to but smaller than the Trapezium cluster (Lada et al. 1991a,b; Lada 1992). The compact young clusters that have been found in both the Orion A and B clouds contain most of the young stars known to be associated with these clouds (Lada et al. 1993); thus, in both the Orion region and the Ophiuchus-Scorpius-Centaurus region, most of the recent star formation has taken place in clusters embedded in dense molecular clumps located at the edges of OB associations. These examples and others, such as the dense cloud cores adjacent to the W4 H II region which also contain embedded young clusters of stars (Lada et al. 1978; Elmegreen 1992), suggest that clusters of stars may often form in massive clumps of molecular gas that have been compressed to particularly high densities by the effects of prior episodes of star formation in their vicinity. The formation of dense clusters may thus represent a type of 'triggered' star formation (Elmegreen \& Lada 1977; Elmegreen 1992), at least in the sense that external compression has caused the stars to form in an exceptionally compact configuration; however, the processes involved are evidently quite complex, and many of the details are not yet clear. The formation of a bound cluster of stars might even be the culminating event in a region of star formation, occurring after several previous episodes of star formation have compressed a small part of the residual gas to a sufficiently high density (Larson 1993). 
Another possibly general feature of star formation in clusters is that most of the cluster stars may form in dense filaments like the OMC1 filament associated with the Trapezium cluster. It may be significant, for example, that the second most prominent newly formed cluster in Orion, the NGC 2024 cluster in the Orion B cloud, also has an associated molecular filament containing a string of dense clumps which appear to be sites of ongoing star formation (Mezger et al. 1992; Mezger, this conference). The NGC 2024 clumps are extremely dense and are also very cold, not yet having been noticeably heated by embedded stars; moreover, they are observed mainly as dust continuum sources and show relatively little molecular line emission, apparently because most of their molecules are frozen out onto dust grains (Mauersberger et al. 1992). These clumps therefore probably represent a very early stage of star formation, possibly even an isothermal phase preceding the appearance of a central stellar object (Mezger et al. 1992); however, at least one of these clumps has already produced a bipolar outflow, indicating that it has already formed or begun to form a central star. Even the $\rho$ Oph cloud contains in the dense $\rho$ Oph A core region a similar chain of very dense and cold clumps which are prominent only in millimeter continuum maps and show little molecular line emission, suggesting that they represent a very early stage of star formation (André, Ward-Thompson, \& Barsony 1993), although once again one of them has already produced a bipolar outflow. The fact that the sites of ongoing star formation in all three of the clusters mentioned are dense clumpy filaments supports the possibility that the fragmentation of filaments is a generally important mechanism of star formation, as discussed in Section 4.

The origin of the filaments that seem to be such ubiquitous features in star-forming regions is not presently understood, but it may be relevant that filamentary structures are a rather general result of gravitational collapse; it was noted in Section 4, for example, that filaments are often produced even in simulations of the collapse of initially spherical clouds. The general tendency for collapsing clouds to become increasingly elongated had in fact been noted much earlier in analytical and numerical studies showing that any initial elongation of a non-spherical cloud tends to be amplified when the cloud collapses gravitationally (Mestel 1965; Lin, Mestel, \& Shu 1965; Larson 1972). However, other effects could also be responsible for the formation of the observed filaments; for example, purely gas-dynamical phenomena such as shear and ablation could play a role, as could helical magnetic fields (Bally et al. 1991; Fukui \& Mizuno 1991). In any case, it is again clear that complex dynamical phenomena must be involved.

\section{Cloud Destruction and Recycling}

Clearly, star formation is highly destructive to molecular clouds; as was emphasized in Section 2, these clouds typically survive for only a few times $10^{7}$ years before being destroyed, or at least restructured in a major way by the effects of star formation. The most effective cloud destruction mechanism may simply be ionization by massive hot stars, which can evaporate away from a star-forming cloud many times the amount of mass that goes into stars; for example, if stars form with a normal mass function, it is only necessary to convert about 4 percent 
of the mass of a cloud into stars to obtain enough hot stars and enough ionizing photons to completely ionize the rest of the cloud (Whitworth 1979; Larson 1987, 1988; Franco 1992). If star formation proceeds continuously in molecular clouds, this implies that its efficiency will only be of the order of 4 percent, since once this fraction of a cloud's mass has been turned into stars, the remainder will soon be ionized and dispersed. The fact that the observed efficiency of star formation in molecular clouds is actually somewhat smaller than this, typically only $\sim 2$ percent or less, may be explainable if stellar winds also contribute importantly to the destruction of these clouds (Leisawitz et al. 1989). Both ionization and winds operate over a period of $\sim 10 \mathrm{Myr}$ or less that is determined by the lifetimes of the most massive stars; this is comparable to the inferred lifetimes of molecular clouds after the onset of star formation in them.

The gas ionized and evaporated from molecular clouds is dispersed in outflows that may often take the form of 'champagne flows' emerging from ionized cavities (Tenorio-Tagle 1979). The total mass flux in these ionized flows is very large, perhaps accounting for as much as half of the mass cycled through molecular clouds in our Galaxy, which was estimated in Section 3 to be at least 150 $\mathrm{M}_{\odot} / \mathrm{yr}$. Ionized outflows from regions of star formation will therefore play an important role in replenishing the warm intercloud component of the interstellar medium. A comparable amount of molecular cloud material may also be dispersed in neutral form by dynamical effects including the pressure exerted by ionized gas, stellar winds, and eventually supernova explosions, and the resulting cloud debris may be swept up into large expanding shells of mostly atomic gas. Such shells are in fact often observed around regions of star formation, including both the Ophiuchus-Scorpius-Centaurus and the Orion regions; a well-known example is Barnard's Loop, which partially surrounds the Orion star-forming region (Blaauw 1991; Bally et al. 1991). The eventual disruption of such shells will replenish the cold neutral component of the interstellar medium, and may in fact be the major source of new interstellar clouds, as was originally suggested by Oort (1954).

The fact that both the ionized and the neutral debris from molecular clouds are predicted to be dispersed with velocities of the order of $10 \mathrm{~km} / \mathrm{s}$, and the fact that gas is observed to be receding from some young clusters with velocities of this order (Leisawitz et al. 1989), strongly suggest that the destruction of starforming clouds is also a major contributor to the observed turbulent motions in the interstellar medium, and that it may largely account for the typical cloud velocity dispersion of $\sim 5$ to $10 \mathrm{~km} / \mathrm{s}$ (Larson 1987; Tenorio-Tagle \& Bodenheimer 1988; Bally et al. 1991). This is possible because the interstellar medium is continually being cycled through star-forming clouds on a timescale that was estimated in Section 3 to be about 50 Myr locally; this cycling time, which is also the time required to regenerate cloud velocities of the order of $10 \mathrm{~km} / \mathrm{s}$, is not much longer than the timescale of $\sim 20$ Myr estimated for the damping of interstellar cloud motions (Spitzer 1978), or the time interval of $\sim 30$ Myr between the collisions of large shells (Bally et al. 1991). Supernova explosions will of course generate additional interstellar cloud motions, but they need not be the dominant source of such motions, as has usually been assumed. Thus, the cycling of interstellar matter through star-forming clouds and the 
subsequent disruption of these clouds by the various effects of star formation may account for many of the observed properties of the interstellar medium.

The cycle of cloud formation and destruction is finally closed when the debris from old star-forming clouds becomes the raw material for building new molecular clouds by the large-scale gravitational instability effects discussed in Section 3. Clearly, many forms of interstellar matter and many types of processes will play a role in the continual cycling of interstellar matter between diffuse and condensed forms that has been discussed. These processes are important not only because of their implications for molecular cloud evolution and star formation, but also because of their broader significance for galactic evolution. One example of the implications of cloud formation and destruction processes for galactic evolution is that they play an important role in the dispersal through the interstellar medium of the heavy elements produced in stars, and hence in the chemical evolution of galaxies (Bateman \& Larson 1993). Thus, the cycle of cloud formation and destruction is a central aspect not only of the evolution of molecular clouds but also of the evolution of galaxies as a whole, and one that has many fundamental implications.

\section{References}

André, P., Ward-Thompson, D., \& Barsony, M. 1993, ApJ, 406, 122

Arcoragi, J.-P., Bonnell, I., Martel, H., Benz, W., \& Bastien, P. 1991, ApJ, 380, 476

Bally, J., Langer, W. D., Stark, A. A., \& Wilson, R. W. 1987, ApJ, 312, L45

Bally, J., Langer, W. D., Wilson, R. W., Stark, A. A., \& Pound, M. W. 1991, in Fragmentation of Molecular Clouds and Star Formation, IAU Symposium No. 147, eds. E. Falgarone, F. Boulanger, \& G. Duvert (Kluwer, Dordrecht), p. 11

Bash, F. N., Green, E., \& Peters, W. L. 1977, ApJ, 217, 464

Bastien, P., Arcoragi, J.-P., Benz, W., Bonnell, I., \& Martel, H. 1991, ApJ, 378, 255

Bateman, N. P. T., \& Larson, R. B. 1993, ApJ, 407, 634

Blaauw, A. 1964, ARA\&A, 2, 213

Blaauw, A. 1991, in The Physics of Star Formation and Early Stellar Evolution, eds. C. J. Lada \& N. D. Kylafis (Kluwer, Dordrecht), p. 125

Blitz, L. 1988, in Millimetre and Submillimetre Astronomy, eds. R. D. Wolstencroft \& W. B. Burton (Kluwer, Dordrecht), p. 269

Blitz, L. 1991, in The Physics of Star Formation and Early Stellar Evolution, eds. C. J. Lada \& N. D. Kylafis (Kluwer, Dordrecht), p. 3

Blitz, L., \& Shu, F. H. 1980, ApJ, 238, 148

Bonnell, I., \& Bastien, P. 1992, ApJ, 401, 654

Bonnell, I., Martel, H., Bastien, P., Arcoragi, J.-P., \& Benz, W. 1991, ApJ, 377, 553

Bonnell, I., Arcoragi, J.-P., Martel, H., \& Bastien, P. 1992, ApJ, 400, 579

Boss, A. P. 1993, ApJ, 410, 157 
Boulanger, F. 1991, in Fragmentation of Molecular Clouds and Star Formation, IAU Symposium No. 147, eds. E. Falgarone, F. Boulanger, \& G. Duvert (Kluwer, Dordrecht), cover photograph

Buat, V., Deharveng, J. M., \& Donas, J. 1989, A\&A, 223, 42

Burkert, A., \& Bodenheimer, P. 1993, MNRAS, in press

Carpenter, J. M., Snell, R. L., Schloerb, F. P., \& Skrutskie, M. F. 1993, ApJ, 407,657

Chièze, J. P., \& Pineau des Forêts, G. 1990, in Physical Processes in Fragmentation and Star Formation, eds. R. Capuzzo-Dolcetta, C. Chiosi, \& A. Di Fazio (Kluwer, Dordrecht), p. 17

Cohen, M., \& Kuhi, L. V. 1979, ApJS, 41, 743

de Geus, E. J. 1991, in The Formation and Evolution of Star Clusters, ed. K. Janes (ASP, San Francisco), p. 40

de Geus, E. J., Bronfman, L., \& Thaddeus, P. 1990, A\&A, 231, 137

Dickman, R. L., Horvath, M. A., \& Margulis, M. 1990, ApJ, 365, 586

Efremov, Y. N. 1989, Soviet Scientific Reviews E: Astrophysics and Space Science Reviews, 7, 105

Elmegreen, B. G. 1989, ApJ, 338, 178

Elmegreen, B. G. 1990a, in The Evolution of the Interstellar Medium, ed. L. Blitz (ASP, San Francisco), p. 247

Elmegreen, B. G. 1990b, ApJ, 357, 125

Elmegreen, B. G. 1991a, in The Physics of Star Formation and Early Stellar

Evolution, eds. C. J. Lada \& N. D. Kylafis (Kluwer, Dordrecht), p. 35

Elmegreen, B. G. 1991b, ApJ, 378, 139

Elmegreen, B. G. 1992, in Star Formation in Stellar Systems, eds. G. TenorioTagle, M. Prieto, \& F. Sánchez (Cambridge University Press, Cambridge), p. 381

Elmegreen, B. G. 1993, ApJ, 411, 170

Elmegreen, B. G., \& Lada, C. J. 1977, ApJ, 214, 725

Evans, N. J. 1991, in Frontiers of Stellar Evolution, ed. D. L. Lambert (ASP, San Francisco), p. 45

Evans, N. J., \& Lada, E. A. 1991, in Fragmentation of Molecular Clouds and Star Formation, IAU Symposium No. 147, eds. E. Falgarone, F. Boulanger, \& G. Duvert (Kluwer, Dordrecht), p. 293

Falgarone, E. 1992, in Astrochemistry of Cosmic Phenomena, IAU Symposium No. 150, ed. P. D. Singh (Kluwer, Dordrecht), p. 159

Falgarone, E., \& Phillips, T. G. 1991, in Fragmentation of Molecular Clouds and Star Formation, IAU Symposium No. 147, eds. E. Falgarone, F. Boulanger, \& G. Duvert (Kluwer, Dordrecht), p. 119

Falgarone, E., Phillips, T. G., \& Walker, C. K. 1991, ApJ, 378, 186

Falgarone, E., Puget, J.-L., \& Pérault, M. 1992, A\&A, 257, 715

Franco, J. 1992, in Star Formation in Stellar Systems, eds. G. Tenorio-Tagle, M.

Prieto, \& F. Sánchez (Cambridge University Press, Cambridge), p. 515

Fukui, Y., \& Mizuno, A. 1991, in Fragmentation of Molecular Clouds and Star

Formation, IAU Symposium No. 147, eds. E. Falgarone, F. Boulanger, \& G.

Duvert (Kluwer, Dordrecht), p. 275

Genzel, R., \& Stutzki, J. 1989, ARA\&A, 27, 41 
Goodman, A. A., Benson, P. J., Fuller, G. A., \& Myers, P. C. 1993, ApJ, 406, 528

Gomez, M., Hartmann, L., Kenyon, S. J., \& Hewett, R. 1993, AJ, 105, 1927

Greene, T. P., \& Young, E. T. 1992, ApJ, 395, 516

Hasegawa, T. 1987, in Star Forming Regions, IAU Symposium No. 115, eds. M. Peimbert \& J. Jugaku (Reidel, Dordrecht), p. 123

Heller, C. H. 1993a, ApJ, 408, 337

Heller, C. H. 1993b,c, ApJ, in press

Herbertz, R., Ungerechts, H., \& Winnewisser, G. 1991, A\&A, 249, 483

Herbig, G. H. 1977, ApJ, 214, 747

Herbig, G. H., \& Terndrup, D. M. 1986, ApJ, 307, 609

Herbst, E., \& Leung, C. M. 1989, ApJS, 69, 271

Hetem, A., \& Lépine, J. R. D. 1993, A\&A, 270, 451

Kennicutt, R. C. 1989, ApJ, 344, 685

Kennicutt, R. C. 1990, in The Interstellar Medium in Galaxies, eds. H. A.

Thronson \& J. M. Shull (Kluwer, Dordrecht), p. 405

Lada, C. J., \& Lada, E. A. 1991, in The Formation and Evolution of Star

Clusters, ed. K. Janes (ASP, San Francisco), p. 3

Lada, C. J., Elmegreen, B. G., Cong, H.-I., \& Thaddeus, P. 1978, ApJ, 226, L39

Lada, E. A. 1992, ApJ, 393, L25

Lada, E. A., Bally, J., \& Stark, A. A. 1991a, ApJ, 368, 432

Lada, E. A., DePoy, D. L., Evans, N. J., \& Gatley, I. 1991b, ApJ, 371, 171

Lada, E. A., Strom, K. M., \& Myers, P. C. 1993, in Protostars and Planets III, eds. E. H. Levy \& J. I. Lunine (University of Arizona Press, Tucson), p. 245

Larson, R. B. 1972, MNRAS, 156, 437

Larson, R. B. 1978, MNRAS, 184, 69

Larson, R. B. 1979, MNRAS, 186, 479

Larson, R. B. 1981, MNRAS, 194, 809

Larson, R. B. 1982, MNRAS, 200, 159

Larson, R. B. 1984, MNRAS, 206, 197

Larson, R. B. 1985, MNRAS, 214, 379

Larson, R. B. 1987, in Starbursts and Galaxy Evolution, eds. T. X. Thuan, T. Montmerle, \& J. Tran Thanh Van (Editions Frontières, Gif Sur Yvette), p. 467

Larson, R. B. 1988, in Galactic and Extragalactic Star Formation, eds. R. E. Pudritz \& M. Fich (Kluwer, Dordrecht), p. 459

Larson, R. B. 1990, in Physical Processes in Fragmentation and Star Formation, eds. R. Capuzzo-Dolcetta, C. Chiosi, \& A. Di Fazio (Kluwer, Dordrecht), p. 389

Larson, R. B. 1991a, in Frontiers of Stellar Evolution, ed. D. L. Lambert (ASP, San Francisco), p. 571

Larson, R. B. 1991b, in Fragmentation of Molecular Clouds and Star Formation, IAU Symposium No. 147, eds. E. Falgarone, F. Boulanger, \& G. Duvert (Kluwer, Dordrecht), p. 261

Larson, R. B. 1992a, MNRAS, 256, 641

Larson, R. B. 1992b, in Star Formation in Stellar Systems, eds. G. TenorioTagle, M. Prieto, \& F. Sánchez (Cambridge University Press, Cambridge), p. 125 
Larson, R. B. 1993, in The Globular Cluster - Galaxy Connection, eds. G. H. Smith \& J. P. Brodie (ASP, San Francisco), p. 675

Lin, C. C., Mestel, L., \& Shu, F. H. 1965, ApJ, 142, 1431

Leisawitz, D., Bash, F. N., \& Thaddeus, P. 1989, ApJS, 70, 731

Mauersberger, R., Wilson, T. L., Mezger, P. G., Gaume, R., \& Johnston, K. J. 1992, A\&A, 256, 640

McCaughrean, M. J. 1989, BAAS, 21, 712; see also Sky and Telescope, 77, 352

McCaughrean, M. 1993, in Massive Stars: Their Lives in the Interstellar Medium, eds. J. P. Cassinelli \& E. B. Churchwell (ASP, San Francisco), p. 80

McCaughrean, M., Rayner, J., \& Zinnecker, H. 1991, Mem. Soc. Astr. Ital., 62, 715

Mestel, L. 1965, QJRAS, 6, 161

Mezger, P. G., Sievers, A. W., Haslam, C. G. T., Kreysa, E., Lemke, R., Mauersberger, R., \& Wilson, T. L. 1992, A\&A, 256, 631

Miyama, S. M., Hayashi, C., \& Narita, S. 1984, ApJ, 279, 621

Miyama, S. M., Narita, S., \& Hayashi, C. 1987a, Prog. Theor. Phys., 78, 1051

Miyama, S. M., Narita, S., \& Hayashi, C. 1987b, Prog. Theor. Phys., 78, 1273

Monaghan, J. J., \& Lattanzio, J. C. 1991, ApJ, 375, 177

Monaghan, J. J., \& Varnas, S. R. 1988, MNRAS, 231, 515

Mooney, T. J., \& Solomon, P. M. 1988, ApJ, 334, L51

Myers, P. C. 1987, in Star Forming Regions, IAU Symposium No. 115, eds. M. Peimbert \& J. Jugaku (Reidel, Dordrecht), p. 33

Myers, P. C. 1991, in The Formation and Evolution of Star Clusters, ed. K. Janes (ASP, San Francisco), p. 73

Myers, P. C., Dame, T. M., Thaddeus, P., Cohen, R. S., Silverberg, R. F., Dwek, E., \& Hauser, M. G. 1986, ApJ, 301, 398

Myers, P. C., Fuller, G. A., Goodman, A. A., \& Benson, P. J. 1991, ApJ, 376, 561

Oort, J. H. 1954, BAN, 12, 177

Prasad, S. S. 1991, in Fragmentation of Molecular Clouds and Star Formation, IAU Symposium No. 147, eds. E. Falgarone, F. Boulanger, \& G. Duvert (Kluwer, Dordrecht), p. 93

Prasad, S. S., Tarafdar, S. P., Villere, K. R., \& Huntress, W. T. 1987, in Interstellar Processes, eds. D. J. Hollenbach \& H. A. Thronson (Reidel, Dordrecht), p. 631

Pringle, J. E. 1991, in The Physics of Star Formation and Early Stellar Evolution, eds. C. J. Lada \& N. D. Kylafis (Kluwer, Dordrecht), p. 437

Rubio, M., Lequeux, J., \& Boulanger, F. 1993, A\&A, 271, 9

Sargent, A. I., \& Mundy, L. G. 1988, in Galactic and Extragalactic Star Formation, eds. R. E. Pudritz \& M. Fich (Kluwer, Dordrecht), p. 261

Scalo, J. 1990, in Physical Processes in Fragmentation and Star Formation, eds.

R. Capuzzo-Dolcetta, C. Chiosi, \& A. Di Fazio (Kluwer, Dordrecht), p. 151

Schneider, S., \& Elmegreen, B. G. 1979, ApJS, 41, 87

Spitzer, L. 1978, Physical Processes in the Interstellar Medium (Wiley, New York)

Sreenivasan, K. 1991, Ann. Rev. Fluid Mech., 23, 539

Stahler, S. W. 1984, ApJ, 281, 209

Tenorio-Tagle, G. 1979, A\&A, 71, 59 
Tenorio-Tagle, G., \& Bodenheimer, P. 1988, ARA\&A, 26, 145

Toomre, A. 1981, in The Structure and Evolution of Normal Galaxies, eds. S. M. Fall \& D. Lynden-Bell (Cambridge University Press, Cambridge), p. 111

Welch, Wm. J. 1993, in Massive Stars: Their Lives in the Interstellar Medium, eds. J. P. Cassinelli \& E. B. Churchwell (ASP, San Francisco), p. 15

Welch, Wm. J., Dreher, J. W., Jackson, J. M., Terebey, S., \& Vogel, S. N. 1987, Science, 238, 1550

Whitworth, A., 1979, MNRAS, 186, 59

Wilking, B. A. 1992, in Low Mass Star Formation in Southern Molecular Clouds, ESO Scientific Report No. 11, ed. B. Reipurth (ESO, Garching), p. 159

Woodward, P. R. 1978, ARA\&A, 16, 555

Williams, J. P., \& Blitz, L. 1993, ApJ, 405, L75

Zimmermann, T., \& Stutzki, J. 1992, Physica A, 191, 79

Zinnecker, H., McCaughrean, M. J., \& Wilking, B. A. 1993, in Protostars and Planets III, eds. E. H. Levy \& J. I. Lunine (University of Arizona Press, Tucson), p. 429 